\title{
On the Amplitude Origin Problem in Dynamical Direct Methods
}

\author{
Wharton Sinkler ${ }^{1}$ and Laurence D. Marks ${ }^{2}$ \\ ${ }^{1}$ UOP LLC, 25 E. Algonquin Rd. Des Plaines, IL 60017-5017, wsinkler@uop.com \\ ${ }^{2}$ Northwestern Universtity, Dept of Materials Science, Evanston, IL 60208-3108
}

Dynamical direct methods (DDM) is the analogue to classical crystallographic direct methods for the case of strongly dynamical diffraction in which the object to be reconstructed is not real-valued but is a complex electron exit wave. This is a new field, and is still in developmental stages. We here describe a basic problem confronting DDM and one possible approach to solving it.

Classical DM combines measurements of diffraction amplitudes, plus the knowledge that the object to which they correspond in real space is mainly flat and $\approx 0$, except at sharp maxima corresponding to atoms. From electron channeling theory, the electron exit wave (specifically $\psi(\mathbf{r})-1$, where the normalized direct beam amplitude is subtracted) also has sharp atom-like peaks. The peaks oscillate in phase as a function of thickness and different peaks oscillate at different rates. A simulated example of this is shown in Fig. 1. This suggests that DDM might succeed as classical DM does using iterative projections onto sets - by turns enforcing the known Fourier amplitudes $\Psi(\mathbf{h})$ in reciprocal space, and implementing a 'sharpening' of $\psi(\mathbf{r})$ after transforming to real space. If successful, iteration would converge to a solution $\psi_{\mathrm{s}}(\mathrm{r}) \approx \psi(\mathbf{r})-1$.

Previous attempts at DDM [1] have used a real space operator given by $\psi(\mathbf{r}) \ln (|\psi(\mathbf{r})| /|<\psi(\mathbf{r})|>)$, in which $<>$ indicates the mean value. This does not alter or depend on the phase of $\psi(r)$ but operates only on the amplitude to perform a sharpening. One difficulty is that one needs to accurately define the zero or background level in the real space domain, which corresponds to knowing the amplitude and phase of the direct beam $\Psi(\mathbf{0})$. If this amplitude origin is badly defined, the peaks may be inverted, and the operator may spuriously blow up areas which should correspond to the background in the correct solution. Severe failure to converge results from this.

In the present work, a novel approach was taken, which is possible if it is assumed that the exit wave is dominated by two discrete phase branches, and that the phases for these branches are known or can be guessed. In strict terms this will correspond to a 2-atom structure (but it may loosely apply to more complex structures if some of the branches have nearly the same phase). In this approach, the amplitude and initial (guessed) phases are transformed to real space, and all the points in the $\psi(\mathbf{r})$ map are projected onto the known phase branches, resulting in two real-valued maps, $a_{1}(\mathbf{r})$ and $a_{2}(\mathbf{r})$. An additional constraint on the wave is that intensity on the two branches does not overlap (if it did, this would result in points at phases which are linear combinations of the branches, which would destroy the 'finger-like' appearance of Fig. 1b). Thus, an origin can be found by minimizing the product $\mathrm{a}_{1}(\mathbf{r}) \times \mathrm{a}_{2}(\mathbf{r})$ using addition of a constant amplitude to each map (in fact $\sum\left(a_{1}(\mathbf{r}) \times a_{2}(\mathbf{r})\right)^{2}$ was minimized). Following the shift of the origin to the proper position, the branches are back-projected to a shifted complex $\psi(\mathbf{r})$, and the real-space sharpening operation is performed.

The availability of two real-valued maps with estimates of the peaks corresponding to the two species in the structure also offers a further interesting possibility of performing the sharpening operation on the two branches separately, followed by back-projection to the complex $\psi(\mathbf{r})$. This reverses the final two steps of the real-space operation, and its effect is to depress regions of overlap (both $\mathrm{a}_{1}(\mathbf{r})$ and $\mathrm{a}_{2}(\mathbf{r})$ large) relative to 
regions of the same amplitude but having less overlap. In addition to sharpening, this thus enforces 'branchedness' of the real-space phase distribution.

Model experiments were carried out on the same structure shown in Fig. 1, in which all cations were replaced by gallium. A strict two-phase channeling wave was calculated as input, and tests were performed for convergence of phase extension. For the phase extensions, varying numbers of strong beams had phases set initially to the correct values (they vary in iteration). Four cases were tested:

1) Origin estimated using $\Psi(\mathbf{0})=\sum \hookrightarrow \Psi(\mathbf{h})^{2}$, operation using $\psi(\mathbf{r}) \ln (|\psi(\mathbf{r})| /<|\psi(\mathbf{r})|>$

2) Origin determined by minimization of $\sum\left(\mathrm{a}_{1}(\mathbf{r}) \times \mathrm{a}_{2}(\mathbf{r})\right)^{2}$, operation using $\psi(\mathbf{r}) \ln (|\psi(\mathbf{r})| /<|\psi(\mathbf{r})|>$

3) Origin as in 1), operation as $\psi(\mathbf{r})=\exp \left(i \phi_{1}\right) \times \mathrm{a}_{1}(\mathbf{r}) \ln \left(\left|\mathrm{a}_{1}(\mathbf{r})\right| /<\mathrm{a}_{1}(\mathbf{r})>+\exp \left(i \phi_{2}\right) \times \mathrm{a}_{2}(\mathbf{r}) \ln \left(\left|\mathrm{a}_{2}(\mathbf{r})\right| /<\mathrm{a}_{2}(\mathbf{r})>\right.\right.$

4) Origin as in 2), operation as $\psi(\mathbf{r})=\exp \left(i \phi_{1}\right) \times \mathrm{a}_{1}(\mathbf{r}) \ln \left(\left|\mathrm{a}_{1}(\mathbf{r})\right| /<\mathrm{a}_{1}(\mathbf{r})>+\exp \left(i \phi_{2}\right) \times \mathrm{a}_{2}(\mathbf{r}) \ln \left(\left|\mathrm{a}_{2}(\mathbf{r})\right| /<\mathrm{a}_{2}(\mathbf{r})>\right.\right.$

Options 2 and 4 define the origin by mimimizing $\sum\left(\mathrm{a}_{1}(\mathbf{r}) \times \mathrm{a}_{2}(\mathbf{r})\right)^{2}$, options 3 and 4 enforce branchedness by reversing the order of sharpening and back-projection, while option 1 is essentially what has been presented previously [1]. Table 1 shows the results of phase extensions for the four options in terms of a figure of merit which compares the result with the correct calculated wave ( 0 is perfect agreement, basic features are generally well-preserved to values of 0.25 ).

Interestingly, no improvement is seen from the new origin definition alone. However, pronounced improvement is found if points of intermediate phase, which are not allowed by the channeling model, are suppressed. This provides proof of principle evidence that enforcement of 'branchedness' may provide a powerful constraint for helping convergence properties in DDM.

\begin{tabular}{|c|c|c|c|c|}
\hline Number & option 1 & option 2 & option 3 & option 4 \\
\hline 12 & 0.260 & 0.418 & 0.174 & 0.400 \\
\hline 18 & 0.223 & 0.399 & 0.107 & 0.135 \\
\hline 24 & 0.138 & 0.199 & 0.112 & 0.094 \\
\hline 30 & 0.135 & 0.182 & 0.076 & 0.093 \\
\hline 36 & 0.079 & 0.080 & 0.020 & 0.049 \\
\hline
\end{tabular}
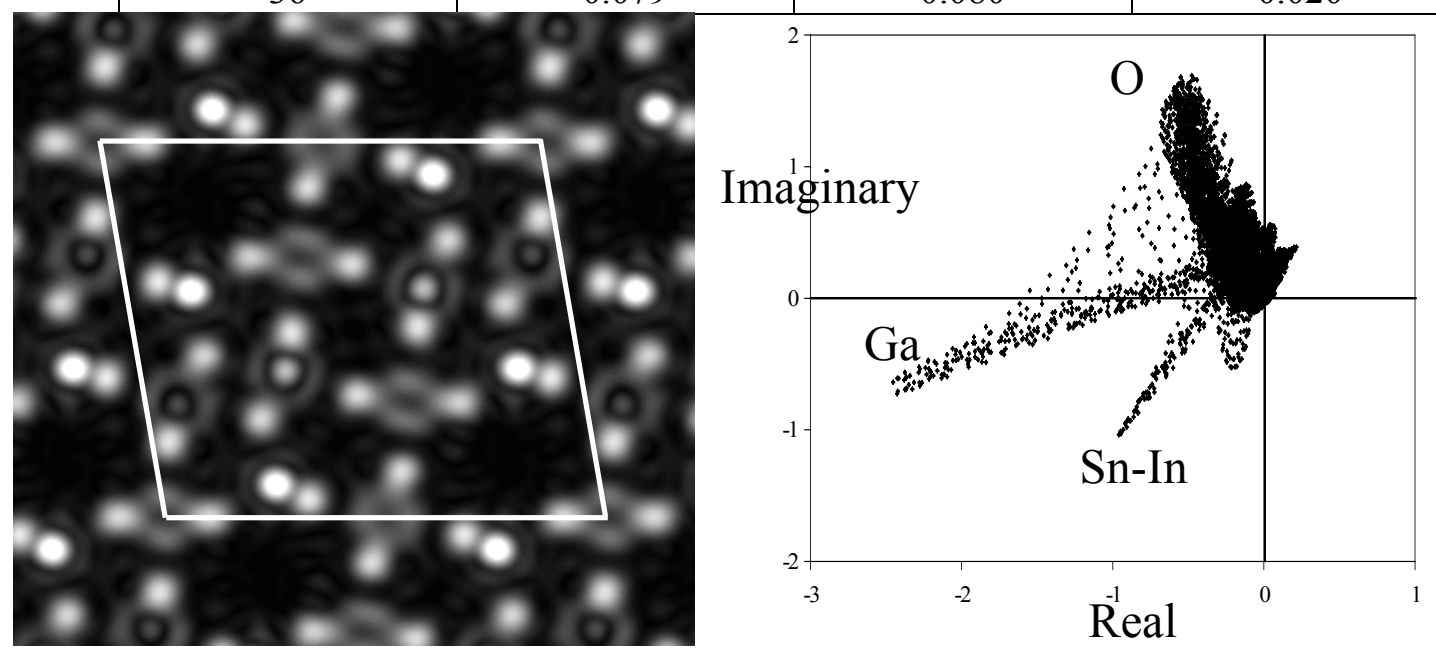

Fig. 1. Calculated wave amplitude (a) and phase (b, as Argand diagram) for $(\mathrm{Ga}, \mathrm{In})_{2} \mathrm{SnO}_{5}$. In $b$, one can see that different peaks of the amplitude reside at distinct phases and correspond to three species of the structure. The 'finger-like' appearance results from little overlap (few intermediate phases).

[1] L. D. Marks and W. Sinkler, submitted to Microscopy and Microanalysis, 2001. 